\title{
Ultraviolet-Bright Type IIP Supernovae from Massive Red Supergiants
}

\author{
Takashi J. Moriya ${ }^{1}$, Nozomu Tominaga ${ }^{2}$, Sergei I. Blinnikov ${ }^{3}$, \\ Petr V. Baklanov ${ }^{3}$, and Elena I. Sorokina ${ }^{4}$ \\ ${ }^{1}$ Kavli IPMU, University of Tokyo, Kashiwanoha 5-1-5, Kashiwa, Chiba 277-8583, Japan \\ email: takashi.moriya@ipmu.jp \\ ${ }^{2}$ Department of Physics, Faculty of Science and Engineering, Konan University, \\ 8-9-1 Okamoto, Kobe, Hyogo 658-8501, Japan \\ ${ }^{3}$ Institute for Theoretical and Experimental Physics, \\ Bolshaya Cheremushkinskaya 25, 117218 Moscow, Russia \\ ${ }^{4}$ Sternberg Astronomical Institute, Moscow University, \\ Universitetski pr. 13, 119992 Moscow, Russia
}

\begin{abstract}
Red supergiants (RSGs) are progenitors of Type IIP supernovae (SNe). It is suggested that RSGs can experience a mass loss with a very high mass-loss rate (even as high as 0.01 $M_{\odot} \mathrm{yr}^{-1}$ ) due to, e.g., dynamical instabilities of their envelopes (e.g., Yoon \& Cantiello (2010)). Because of the extensive mass loss, RSGs can have very dense circumstellar medium (CSM) around them. If a SN explosion occurs soon after the extensive mass loss of a RSG, the SN ejecta will collide with the dense CSM. Due to the collision, the kinetic energy of the ejecta is converted to radiation energy and such SNe with collision can be brighter than usual Type IIP SNe. By performing one-dimensional multi-group radiation hydrodynamical calculations, we investigate the effects of the collision on Type IIP SN LCs. We show that if RSGs explode within a dense CSM, the SN will be very bright, especially in ultraviolet, at early epochs. We also compare our models with the ultraviolet-bright Type IIP SN 2009kf and show that the progenitor of SN $2009 \mathrm{kf}$ can be a massive RSG which experienced extensive mass loss just before its explosion. We conclude that this is evidence that massive RSGs experience extensive mass loss and the existence of such mass loss can actually be the cause of the contradiction between theoretical and observational mass ranges of Type IIP SN progenitors.
\end{abstract}

Keywords. circumstellar matter, stars: mass loss, supernovae: general, supernovae: individual (SN 2009kf)

\section{Introduction}

SN 2009kf is a Type IIP supernova (SN) discovered by the Pan-STARRS 1 survey (Botticella et al. (2010)). The bolometric luminosity of the plateau obtained by just adding the optical light curves (LCs) exceeded $4 \times 10^{42} \mathrm{erg} \mathrm{s}^{-1}$ and SN 2009kf is one of the most luminous Type IIP SNe. It was also observed by the GALEX satellite in the NUV band at the early epochs. Surprisingly, the NUV luminosity stayed almost constant for about 10 days during the GALEX observations. Ultraviolet (UV) LCs of usual Type IIP SNe decline rapidly due to the adiabatic cooling of the SN ejecta after the shock breakout. The very slow decline of UV luminosity observed in one of the most luminous Type IIP, SNe SN 2009kf, is difficult to interpret only with the adiabatic cooling of SN ejecta. 


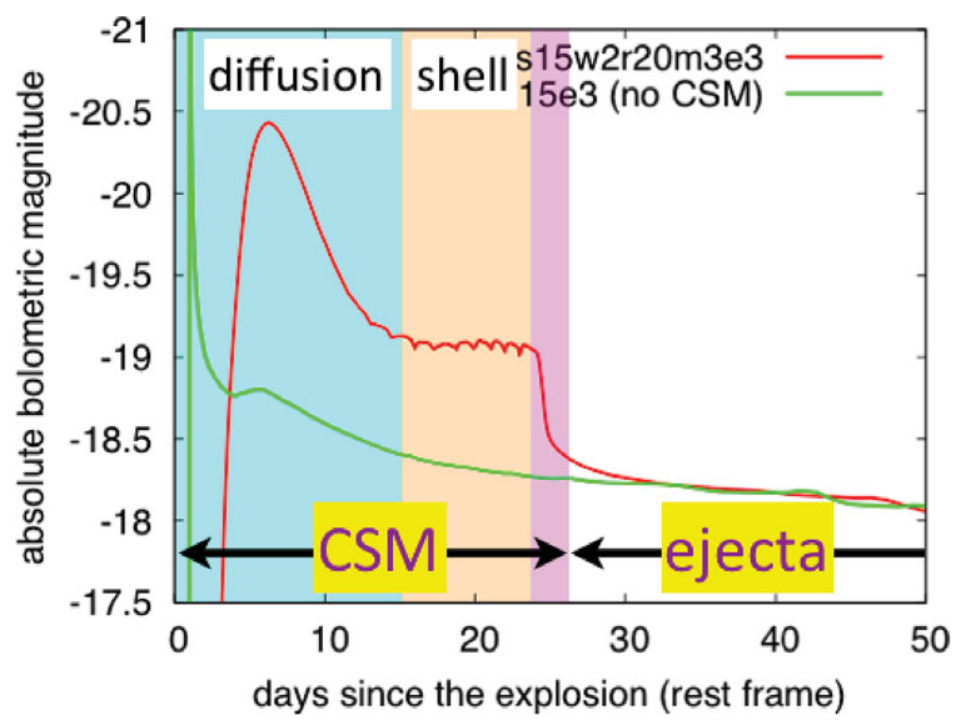

Figure 1. Early LCs from the explosion of an RSG with and without dense CSM.

\section{UV-Bright Type IIP SNe}

One possible cause of the slowly-declining high UV luminosity is the existence of a dense circumstellar medium (CSM) around the progenitor. With the typical mass loss history of RSGs, we do not expect the existence of a dense enough CSM around RSGs to affect the optical LCs of the subsequent SN. However, some RSGs are observationally known to have much higher mass-loss rates (e.g., VY CMa (Smith et al. (2009)), IRAS 05280-6910 (Boyer et al. (2010))) and it is also theoretically suggested that some RSGs experience extensive mass loss just before their explosions (e.g., Yoon \& Cantiello (2010)). We investigate the effect of the dense CSM around the progenitors of RSGs on their LCs.

As is shown in Moriya et al. (2011) and Figure 1, the dense CSM affects the early LCs of the explosions of RSGs. The models without RSGs show a quick rise in the LCs because of the shock breakout. If there is a dense CSM around the progenitor, however, the shock breakout signal is extended because of the diffusion in the dense CSM. In addition, because of the interaction, the LC becomes brighter than those without the dense CSM, especially in UV.

Looking into the explosions with dense CSM, the photosphere is located in the CSM and photons diffuse in the CSM at first. Then, the photosphere stays in the cold dense shell between the dense CSM and SN ejecta for a while and the luminosity stays constant at these epochs. When the shock goes through the dense CSM or the remaining CSM becomes small enough, the LC suddenly drops and the effect of the CSM on it is no longer apparent.

The UV-bright LC of SN 2009kf is found to be consistent with an explosion of an RSG with $3 \times 10^{51} \mathrm{erg}$ within a dense CSM from $10^{-2} M_{\odot} \mathrm{yr}^{-1}\left(0.6 M_{\odot}\right), 2 \times 10^{15} \mathrm{~cm}$ (Moriya et al. (2011)). Unfortunately, the spectra of SN 2009kf were only taken during the plateau phase long after the UV-bright phase and all the dense CSM is presumed to be shocked away at these epochs. We expect that if we take the spectra of SN 2009kf-like SNe during the UV-bright phase, the spectral type would be Type IIn, which shows the 
narrow lines due to the dense CSM, and then transforms to Type II spectral type without the effect of the dense CSM.

\section{Implications}

The plateau luminosity of SN $2009 \mathrm{kf}\left(\simeq 4 \times 10^{42} \mathrm{erg} \mathrm{s}^{-1}\right)$ is one of the brightest among the currently known Type IIP SNe. This high luminosity and the very large line velocities obtained from the spectra indicate that SN 2009kf is one of the most energetic explosions of RSGs currently observed. There are known empirical implications that more massive RSGs explode with higher energy (e.g., Hamuy (2003)). If this is true, the progenitor of SN 2009kf is one of the most massive RSGs and close to the upper mass limit of Type IIP SN progenitors. The fact that the progenitor of a Type IIP SN is close to the upper mass limit indicates that the upper mass limit of Type IIP SNe may be determined by the extensive mass loss which is currently not known well. This kind of extensive mass loss by massive RSGs can reduce the upper mass of Type IIP SN progenitors and will be a key to solve the so called 'red supergiant problem' (Smartt et al. (2009)).

\section{References}

Botticella, M. T., et al. 2010, ApJ (Letters), 717, L52

Boyer, M. L., et al. 2010, A\&\&A (Letters), 518, L142

Hamuy, M. 2010, ApJ , 582, 905

Moriya, T., et al. 2011, MNRAS, 415, 199

Smartt, S. J., et al. 2009, MNRAS, 395, 1409

Smith, N., et al. 2009, AJ, 137, 3558

Utrobin, V. P., et al. 2010, ApJ (Letters), 723, L89

Yoon, S.-C. \& Cantiello, M. 2010, ApJ (Letters), 717, L62

\section{Discussion}

KATZ: Can you separate the effects of UV and total brightness due to the two changes: energy and CSM?

MORIYA: The UV LC of the model without CSM with $3 \times 10^{51} \mathrm{erg}$, which is already rather a high explosion energy, is more than two magnitudes fainter than that of SN 2009kf. You can increase the UV luminosity by increasing the energy but then the duration of the UV LC will also decrease and it will be difficult to explain the long duration of the UV LC of SN 2009kf. If you also increase the RSG radius, you can get the LC of SN $2009 \mathrm{kf}$ without CSM but the required values are extreme $\left(2000 R_{\odot}\right.$ and $2.2 \times 10^{52} \mathrm{erg}$, Utrobin et al. (2010)).

Chakraborti: Do you predict X-ray emission?

MORIYA: No, because I expect X-rays are absorbed by the massive CSM.

Chakraborti: Did you observe SN 2009kf, Stefan?

IMMLER: No, I didn't. 
Sмiтh: A progenitor mass-loss rate of $10^{-2} M_{\odot} \mathrm{yr}^{-1}$ is very high, probably requiring an explosive pre-SN outburst instead of a wind. We would usually expect that to make a Type IIn spectrum. Do you have early-time spectra during the CSM-interacting phase to show that SN 2009kf was not a Type IIn supernova?

MORIYA: We do expect that the early time spectra will be those of Type IIn. We expect that the spectral type changes from Type IIn to Type II when the entire dense CSM is shocked away. Unfortunately, the early time spectra of SN 2009kf were not taken. 